\title{
EL IMPACTO DE LA RELACIÓN CON EL CLIENTE Y DE LA CAPACIDAD DE VALOR AGREGADO EN EL SERVICIO EN EL RENDIMIENTO DE LA PYME MANUFACTURERA EN AGUASCALIENTES
}

\author{
The Impact of customer relationship and the ability to value-added service in the performance of \\ manufacturing SMEs in Aguascalientes \\ Gabriela Ortiz-Delgadillo \\ Maestría en Mercadotecnia. Universidad Politécnica de Aguascalientes, Aguascalientes- México \\ gabriela.ortiz@upa.edu.mx \\ Edgar Omar Esquivel-Aguilar \\ Licenciado Administración y Negocios. Universidad Politécnica de Aguascalientes. Aguascalientes- México \\ edgar.aguilar34@hotmail.com

\section{Octavio Hernández-Castorena} \\ Doctor en Ciencias Administrativas. Universidad Autónoma de Aguascalientes, Aguascalientes- México \\ ohernandez@correo.uaa.mx
}

\section{Cómo citar / How to cite}

Ortiz-Delgadillo, G., Esquivel-Aguilar, E. y Hernández-Castorena, O. (2016). El impacto de la relación con el cliente y de la capacidad de valor agregado en el servicio en el rendimiento de la Pyme manufacturera de Aguascalientes. Revista CEA, 2(4), 47-58.

Recibido: 2 de marzo de 2015

Aceptado: 15 de mayo de 2016

\section{Resumen}

La presente investigación se realizó con el objetivo de conocer el impacto de las relaciones con el cliente y la capacidad de agregar valor al servicio sobre el rendimiento de la gestión de la cadena de suministro de las Pymes del estado de Aguascalientes. Para esto, se realizó un estudio cuantitativo, con alcance correlacional, de corte transversal, en el cual se construyó un instrumento de medición tipo escala de Likert. Este instrumento, se encuentra compuesto por 27 ítems, dividido en 3 bloques principales: Relación con los clientes (16 ítems), capacidad de agregar valor agregado al servicio (5 ítems) y rendimiento (6 Ítems), los cuales fueron aplicados a una población de 213 empresarios de la ciudad de Aguascalientes. El análisis de los datos fue realizado a través de la técnica estadística de la regresión lineal, para lo cual se hizo uso del software estadístico IBM SPSS versión 20, donde los resultados más significativos mostraron que la relación con los clientes es parte fundamental para que las
Pymes alcancen un mayor rendimiento en la gestión de la cadena de suministro.

Palabras clave: relación con el cliente, valor agregado, rendimiento, cadena de suministro y Pyme manufacturera.

\begin{abstract}
This research was conducted to know the impact of customer relationships and the ability to add value to the service on the performance of the Management Supply Chain SMEs in the State of Aguascalientes. For this, a quantitative study was conducted with correlational scope, crosssectional, in which an instrument Likert scale Such measurement is built, is composed of 27 items, divided into 3 main blocks: Relationship with customers (16 items), ability to add valueadded service (5 items) and performance ( 6 items). Which they were applied to a population of 213 entrepreneurs in the city of Aguascalientes. The data analysis was performed through the statistical technique of linear
\end{abstract}


El impacto de la relación con el cliente y de la capacidad de valor agregado en el servicio en el rendimiento de la PYME manufacturera

regression, for which made use of statistical software SPSS version 20, where the most significant results showed that the relationship with customers is fundamental to the SMEs to achieve higher performance in managing the supply chain.

Keywords: customer relationships, added value, performance, supply chain and manufacturing SMEs.

\section{INTRODUCCIÓN}

Tomando en cuenta la creciente competitividad económica en el mundo, el dinamismo del mercado y los fenómenos producidos por la globalización, las empresas, en especial las Pyme, se encuentran con diversos retos que les exigen a mejorar e innovar sus procesos comerciales, productivos, logísticos y de abastecimiento, con el fin de implementar estrategias que les permitan cumplir los estándares de calidad y los requerimientos del mercado actual, orientando sus esfuerzos a maximizar el rendimiento general de la cadena de suministro (Gómez, 2008). Dada esta tendencia es que la gestión de la cadena de suministro se destaca como una de los objetos de estudio que revisten gran importancia para la administración moderna (Oly, 2005).

En este contexto, y debido a los constantes cambios del mercado y las tendencias de consumo, es que las empresas encuentran un gran valor en el conocimiento y la información que puedan adquirir sobre las necesidades de los clientes, ya que, al conocer estas necesidades, las empresas podrán desarrollar productos 0 servicios en función de ellas, con lo cual se podrán establecer relaciones a largo plazo con los clientes, basadas en la confianza y el beneficio mutuo.

Si bien es cierto que mantener buenas relaciones con los clientes es parte fundamental del desarrollo de cualquier organización, no se debe olvidar que del otro lado de la moneda se encuentra la necesidad de crear valor en los procesos internos y de esta manera permitir mejorar el rendimiento de la compañía, a través de la creación de alguna ventaja comparativa que lo diferencie de la competencia y a su vez la convierta en una mejor opción para los clientes.

Dada esta situación, la medición del rendimiento de las organizaciones se está convirtiendo cada vez más en un elemento de competitividad y de diferenciación entre aquellas empresas capaces de realizarlo y aquellas otras que no. Es por eso que las empresas, ya sean pequeñas o grandes, tienen como objetivo maximizar la creación de valor mientras se minimizan los costos (Castro, 2008).

Por ello, es importante establecer un modelo que permita identificar las prácticas que una Pyme debe de implementar para satisfacer a sus clientes. En este sentido, algunos de los factores que son tomados en cuenta por los clientes a la hora de elegir una empresa que los provea de productos o servicios son: los tiempos de entrega del producto, la capacidad productiva, la flexibilidad y el manejo de conflictos, la calidad, la posición competitiva, entre otros.

Para esto, las Pymes deben de estar conscientes que el éxito en el trabajo de producción y comercialización depende de una labor colaborativa entre los distintos entes involucrados en el desarrollo de los productos o servicios a ofrecer (Luna, 2009). Sin embargo, la competitividad entre las compañías está pasando de ser un problema local o particular a un problema conjunto de todas las empresas que participan en la cadena de suministro de un producto o servicio; es por eso que Giannakis y Croom, (2004) mencionan que en la actualidad las que compiten no son las empresas sino sus cadenas de suministro.

En este sentido, poner a disposición del mercado productos o servicios de calidad que cumplan con los estándares competitivos actuales, solo será posible si estos han pasado por procesos de excelencia a lo largo de toda la cadena de suministro. Es ahí donde se llevan a cabo los procesos claves del negocio, que van desde los 
proveedores hasta el usuario final $y$ proporcionan productos, servicios e información que agrega valor a los clientes y demás implicados.

La investigación realizada busca determinar el impacto que tienen las relaciones con el cliente y la capacidad de generar valor sobre las prácticas y estrategias que las Pymes del estado de Aguascalientes aplican dentro de la gestión de la cadena de suministro. El presente artículo consta además del marco teórico que sustenta la investigación, de los resultados de la aplicación de la regresión lineal al modelo propuesto, ofreciéndose al final las conclusiones.

\section{MARCO TEÓRICO}

El entorno económico actual se caracteriza por la creciente complejidad de las organizaciones y su entorno, en las cuales el constante cambio en los gustos y hábitos de los consumidores ha producido un desequilibrio en la demanda de los productos ofrecidos, donde factores como la innovación, la competitividad y la calidad han marcado tendencia sobre la dirección estratégica que siguen las empresas en busca de satisfacer las nuevas expectativas del mercado.

Es por esta razón que las empresas se ven exigidas a buscar nuevas formas de generar estrategias y procesos que optimicen la función de la cadena de suministros (García, 2006), a fin de poder ofrecer a sus clientes beneficios que otras empresas no puedan brindarles.

A continuación, para facilitar el entendimiento de esta investigación se presentan algunos de los cuestionamientos más comunes en torno a las variables de estudio.

\section{La cadena de suministro}

Una cadena de suministro es aquella que se encuentra conformada por todas aquellas partes involucradas de manera directa o indirecta en la satisfacción de una solicitud de un cliente. La cadena de suministro incluye no solamente al fabricante y al proveedor, sino también a los transportistas, almacenistas, vendedores al detalle (o menudeo) e incluso a los mismos clientes (Chopra, 2008). A continuación, en la Figura 1, se podrá observar el proceso de la cadena de suministro.

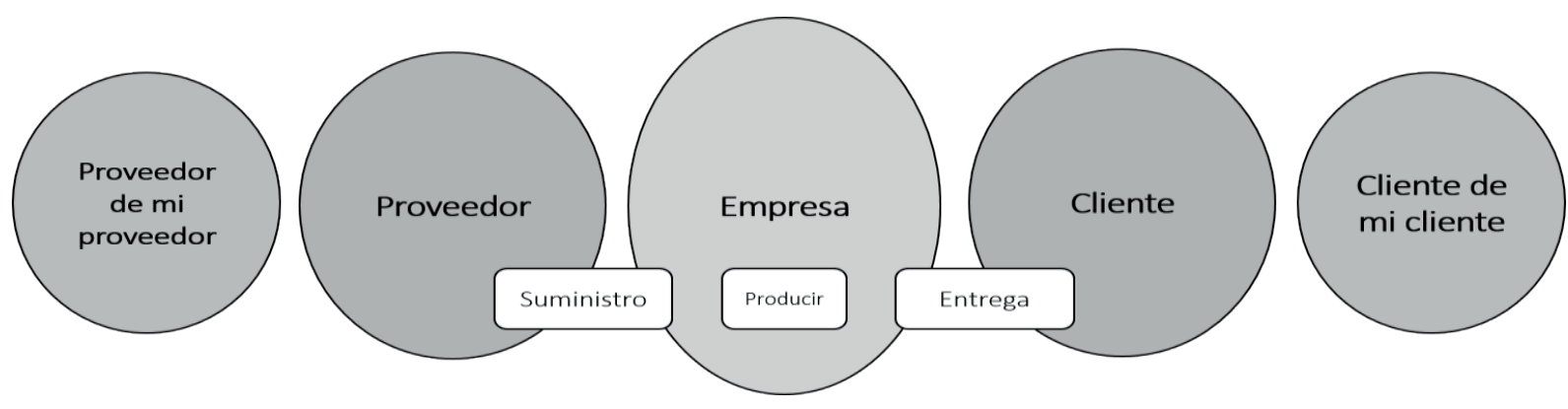

Figura 1. Proceso de la cadena de suministro

Figure 1. Supply Chain Process

Fuente: elaboración propia a partir Gómez, M. G. S. (2008).

No obstante, lo habitual en la mayoría de los casos es que las distintas empresas que componen las cadenas de suministro no establezcan más contacto entre ellas que el estrictamente necesario para la buena marcha de la producción, comunicación de plazos de entrega, problemas surgidos, etc., por lo que no se realiza en ningún momento una coordinación 
El impacto de la relación con el cliente y de la capacidad de valor agregado en el servicio en el rendimiento de la PYME manufacturera

real entre ellas ni un verdadero intercambio de conocimiento (Capó, 2007).

Sin embargo, en el caso particular de las Pyme, para que se produzca una interacción positiva entre las distintas empresas, de forma que se consiga generar un proceso de creación e intercambio de conocimiento, es necesario que se cumplan una serie de requisitos, los cuales necesitan de una similitud entre sistemas de gestión, cultura, lenguaje, objetivos, etc., que no siempre se dan entre las empresas que se relacionan (Capó et al., 2005).

Ahora bien, el concepto puede ser la parte más sencilla de comprender, pero el verdadero reto está en que las empresas reconozcan su valor y los beneficios de saber implementar y gestionar la cadena de suministros, sobre todo en el contexto de las Pymes, las cuales, debido a la poca experiencia suelen cometer errores que en múltiples ocasiones provocan rupturas comerciales derivando en el fracaso de las mismas.

Según Chopra y Meindl (2008), una cadena de suministro está formada por todas aquellas partes involucradas de manera directa o indirecta en la satisfacción de una solicitud de un cliente. Incluye no solamente al fabricante y al proveedor, sino también a los transportistas, almacenistas, vendedores al detalle (o menudeo) e incluso a los mismos clientes. Una cadena de suministro es un grupo de empresas que interactúan dentro y entre sí, con el objetivo de satisfacer a un cliente a través de un producto o un servicio (Torres, 2016).

La creación de valor procede de explotar tanto los vínculos existentes entre procesos y actividades desarrollados en la propia empresa, como los que existen entre las cadenas de valor de sus proveedores y clientes (Gonzálvez, 2016). La utilidad relativa a la posesión se refiere a entregar los bienes en las manos del cliente correcto (Coyle et al., 2003).

Los clientes se evalúan en función de sus demandas o necesidades actuales y futuras, incluyendo el uso que se pretende, características de los diseños deseados, cantidades que se anticipan, preferencias en cuanto a la ubicación geográfica, tiempos de entrega y los tipos de servicios necesarios para incrementar las actividades. Los competidores se evalúan con el fin de determinar su posicionamiento para competir efectivamente. La complejidad del proceso está definida por la manera como el cliente desea utilizar los bienes. Por ejemplo, rubros para la reventa, que pueden ser importantes, generalmente imponen menores riesgos que productos cuyo fin es utilizarse en subsiguientes procesos de manufactura, especialmente si se trata de enfoques de manufactura justo-a-tiempo (Young y Esqueda, 2005).

\section{Importancia de la cadena de suministro}

La gestión efectiva de la cadena de suministro permite una mejor prestación de servicio al cliente y de la cadena de valor. Dicha gestión permite competir con éxito en los mercados actuales, gracias a la implantación de mejores prácticas en sus diferentes áreas; es por eso que, actualmente, se le considera un elemento clave para la competitividad de las empresas, debido a la importancia que tiene en los resultados empresariales a través del margen de beneficio, la calidad de los productos y servicios, la satisfacción del cliente y los plazos de entrega (Stadtler, 2005). 
Etapa 1

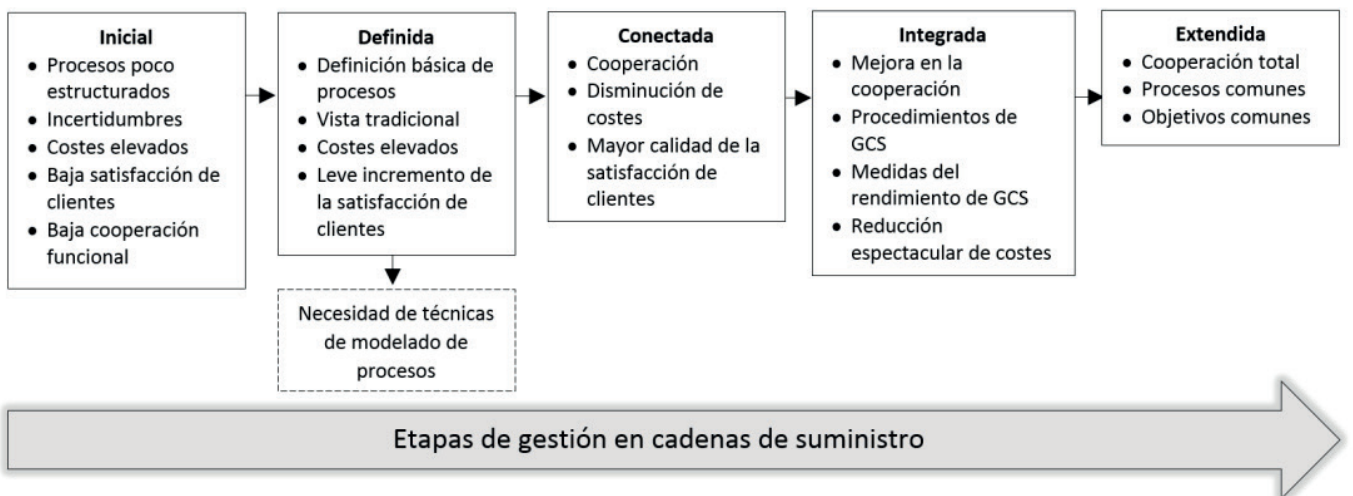

Figura 2. Etapas de maduración de la cadena de suministros

Figure 2. Maturational stages of the supply chain Fuente: Sanchis, Poler y Ortiz (2009).
En este sentido, y poniendo el ejemplo de una Pyme, para que estas logren gestionar correctamente la cadena de suministro deben de atravesar diversas etapas de maduración dentro de sus procesos. En la Figura 2 se podrán visualizar las etapas mencionadas.

\section{La importancia que tiene en el rendimiento de la cadena de suministros las relaciones con los clientes}

El primer paso en la integración de la administración de la cadena de suministro es identificar a los clientes o grupo de clientes que pueden ser considerados como críticos o importantes en la misión comercial de la empresa Jiménez (2002). Es en este punto donde se pueden negociar los acuerdos específicos del servicio, en el cual queden establecidos los tiempos de entrega, la flexibilidad y la respuesta a los problemas generados dentro del proceso, el uso y manejo de los productos o servicios etc. Por lo tanto, precisar y conocer a fondo a sus clientes buscando comprender sus necesidades presentes y futuras es parte fundamental para el establecimiento de relaciones personalizadas con los mismos (Garrido, 2008), ya que satisfacer las necesidades de los clientes es la clave de los intercambios entre las empresas y el mercado (Saura, 2006).
Tomando en cuenta lo anterior, se puede establecer que las relaciones con los clientes se han convertido en una herramienta clave para la gestión de la cadena de suministro y el desarrollo de las empresas, ya que como lo menciona Hashimura (2011), una vez conociendo la importancia de establecer relaciones estrechas con el cliente es que se pueden obtener beneficios, como generar más ingresos, reducir costes y mejorar el retorno sobre la inversión, o como mejor se entiende, el rendimiento de la empresa.

Por su parte, Correa (2009) menciona que establecer buenas relaciones con el cliente mejora la gestión de la de la cadena de suministro, debido a que permite conocer información acerca de las necesidades y la manera de satisfacer a los clientes, lo cual puede mejorar la estimación de la demanda. Ahora bien, un punto a considerar es que las empresas juegan dos papeles importantes dentro de la cadena de suministros, uno de ellos es el de cliente y el otro es el de proveedor.

Zutshi y Creed (2009) plantean que la construcción y la gestión de relaciones clienteproveedor se reconocen hoy como uno de los pilares fundamentales para la creación de ventajas competitivas sostenibles. Por lo que Jae-Eun (2008) afirma que una empresa no compite sola, pues forma parte de una red 
El impacto de la relación con el cliente y de la capacidad de valor agregado en el servicio en el rendimiento de la PYME manufacturera

en Aguascalientes

dentro de la cual debe competir en forma colaborativa, con miras a mejorar su calidad, entrega y rendimiento, mientras que simultáneamente reduce sus costos, todo esto enfocado a posicionar a la empresa en un alto nivel competitivo, además de brindar un mejor servicio para sus clientes.

Sin embargo, seleccionar proveedores que cuenten con las características específicas que se requieren para dar abasto a la empresa, no es tarea sencilla, ya que dentro de la estrategia de aprovisionamiento se debe considerar el impacto de los proveedores en el alcance de los objetivos generales de la organización (Amid, 2006).

\section{La importancia de la capacidad de generar valor en el rendimiento de la cadena de suministros}

La importancia de la capacidad de generar valor en el rendimiento de la cadena de suministro se determinada desde el punto de vista en el cual ambas se completan, ya que como lo menciona Chopra (2008) el objetivo de una cadena de suministro debe ser maximizar el valor total generado, donde para la mayoría de las cadenas de suministro, el valor estará estrechamente correlacionado con la rentabilidad de la misma.

El modelo competitivo actual asume que toda empresa tiene una propuesta de valor para atraer y retener clientes (Calderón, 2006). En este sentido, las empresas, para sobrevivir en el entorno competitivo actual deben centrarse en la consecución de una ventaja competitiva sostenible, basada en el conocimiento, que las diferencie de su competencia. Para lograr dicha diferenciación, las empresas deben de ser capaces de generar valor al cliente durante alguno de los procesos de la cadena de suministro.

Tomando en cuenta que uno de los objetivos principales de cualquier organización es generar valor que lo distinga de la competencia, ya sea en sus productos o servicios, es que se plantean diversas estrategias para lograrlo. Monge (2010) señala que las más importantes son:
- Estrategias competitivas: enfocadas a maximizar el valor de las capacidades y recursos que distinguen a la empresa de sus competidores con el objetivo de crear valor para los compradores.

- Estrategia de liderazgo en costos: enfocada en lograr un coste final mínimo respecto a la competencia, junto con una calidad aceptable y una política de precios que permitan alcanzar un volumen de ventas y un crecimiento de la cuota de mercado rentables.

- Estrategia de diferenciación: enfocada en ofrecer productos o servicios que el mercado perciba como únicos en algunos de los atributos que lo definen.

Finalmente, se puede establecer que el rendimiento de la cadena de suministro basa su éxito en la consecución de algún valor que pueda ser percibido por los clientes.

\section{METODOLOGÍA}

El presente trabajo de investigación se planteó con el objetivo de conocer el impacto de las relaciones con el cliente y la capacidad de agregar valor al servicio, sobre el rendimiento de la gestión de la cadena de suministro de las Pymes del estado de Aguascalientes. Esta es una investigación cuantitativa, con alcance correlacional, de corte transversal; para ello se contó con una población de 213 empresarios de la ciudad de Aguascalientes. El instrumento utilizado para la recolección de datos corresponde a una encuesta formada por 27 ítems, medida a través de la escala de Likert 1-5, la cual se encuentra dividida en 3 bloques: relación con los clientes (16 ítems), capacidad de agregar valor al servicio (5 ítems) y rendimiento (6 Ítems); mientras que el análisis de los datos fue realizado a través de la técnica estadística de la regresión lineal y el análisis factorial, con apoyo del software estadístico SPSS v20; para ello se plantean las siguientes hipótesis: 
$\mathrm{H} 1$ : la relación con los clientes es la variable que más influye al rendimiento de la cadena de suministro

H2: a mejor relación con los clientes mayor rendimiento de la cadena de suministro
H3: las Pymes en Aguascalientes dan una gran importancia a la generación de valor en el servicio

H4: las Pymes en Aguascalientes dan una gran importancia a las relaciones con el cliente.

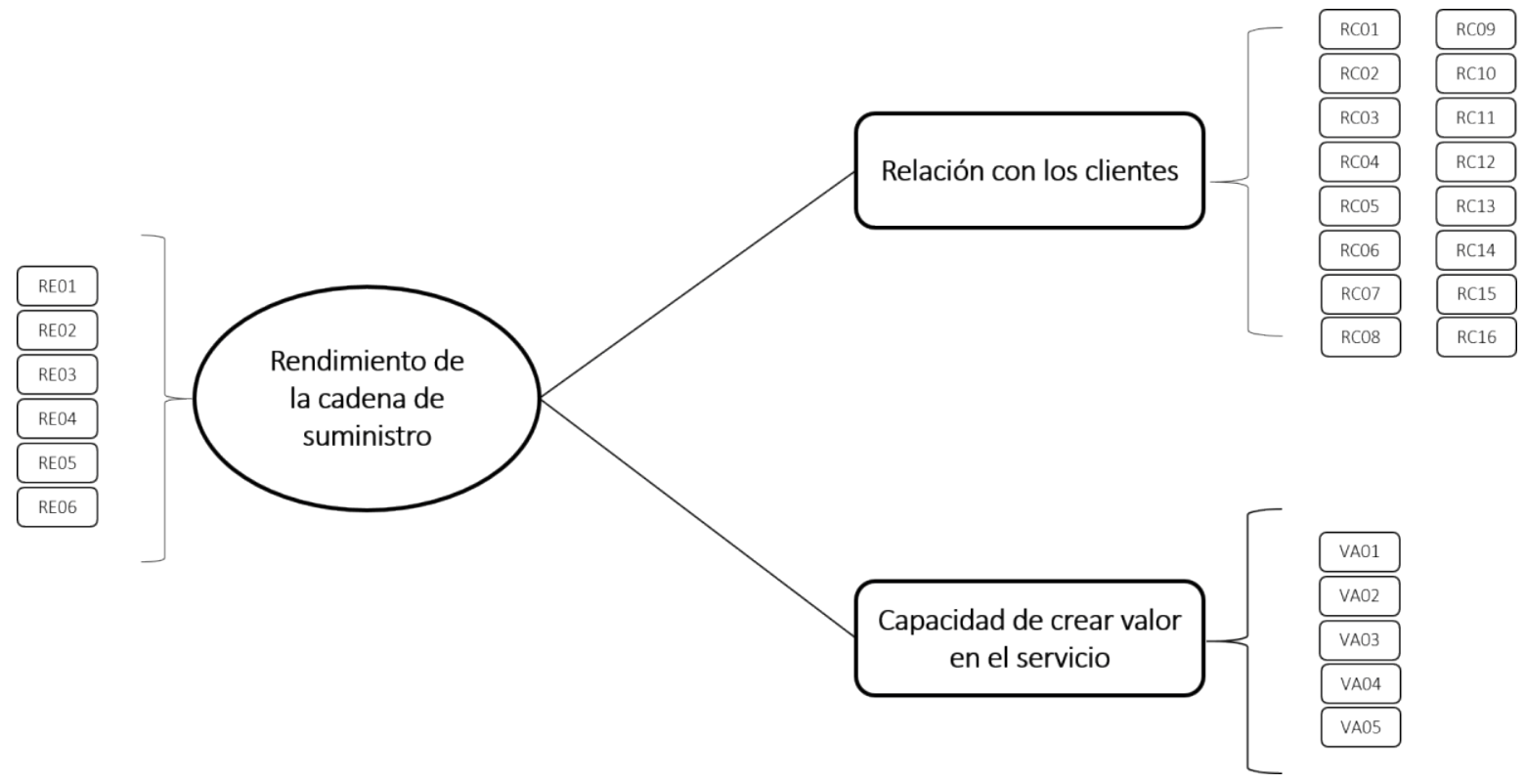

Figura 3. Modelo de la investigación

Figure 3. Model of research

Fuente: elaboración propia.

\section{RESULTADOS}

\section{Resultados del primer modelo rendimiento y relación con el cliente}

Para comenzar con los resultados de la presente investigación, se realizó un análisis mediante el uso de la técnica estadística Regresión lineal, bajo el método de pasos sucesivos, a través del software estadístico SPSS V20, con el objetivo de probar las hipótesis de investigación. A continuación, en la Tabla 1 se muestran los resultados obtenidos en el primer modelo, donde R obtuvo un valor de 0.995, una R2 de 0.991 y una R2 corregida de 0.991, por lo que se establece que la relación con el cliente se encuentra correlacionado con el rendimiento en un 99.5\%, mientras que la influencia ejercida por parte de las relaciones con el cliente corresponde al 99.1\%, con lo cual a su vez se explica la estructura del modelo teórico, así mismo, se puede concluir que la variable relaciones con el cliente influye de manera significativa al desempeño, con un valor en significancia de 0.000 , mientras que el valor FIV (Factor de Inflación de Varianza) mostró un resultado de 1.000, por lo que se establece que el modelo no tiene problemas de multicolinealidad, ya que el valor se encuentra cercano a la unidad (Hair et al. 2006). 
El impacto de la relación con el cliente y de la capacidad de valor agregado en el servicio en el rendimiento de la PYME manufacturera en Aguascalientes

Tabla 1. Resumen del modelo rendimiento-relación con el cliente

Table 1. Model Summary performance-customer relationship

\begin{tabular}{lc}
\hline \multicolumn{1}{c}{ Modelo 1 } & Valores \\
\hline$R$ & 0.995 \\
R cuadrado & 0.991 \\
R cuadrado corregida & 0.991 \\
Error tip. de la estimación & .63291 \\
Durbin y Watson & 2.007 \\
Alfa de Cronbach & 0.961 \\
FIV & 1.000 \\
Significancia & 0.000 \\
\hline
\end{tabular}

Fuente: elaboración propia a partir de los resultados obtenidos del software SPSS versión 20 .

El análisis bivariado, efectuado mediante el cálculo de correlación de Pearson, arrojó asociaciones significativas en todos los ítems considerados en la variable relación con el cliente sobre el rendimiento. Las correlaciones que más se destacan en este apartado son: «Capacidad de cumplir con las fechas que los clientes requieren» $(\beta=0.993) \quad\left(P<0.005^{* *}\right)$, «La flexibilidad en las necesidades cambiantes de los clientes» $(\beta=0.992) \quad\left(P<0.005^{* *}\right)$, «La determinación de los factores clave para mejorar la satisfacción del cliente» $(\beta=0.992)$ ( $P$ $\left.<0.005^{* *}\right)$, «El tiempo de entrega de productos directamente a los clientes» $(\beta=0.991)$, entre otros.

Algo que también debe tenerse en consideración, es el resultado obtenido en el coeficiente alfa de Cronbach, en el que se obtuvo un resultado de 0.961, con lo cual se puede establecer que los niveles de fiabilidad del instrumento son altos y por ende están en un nivel óptimo para los propósitos de la investigación, ya que como lo menciona Celina y Campo (2005), el valor mínimo aceptable para el coeficiente alfa de Cronbach es 0.7; por debajo de ese valor la consistencia interna de la escala utilizada es baja.

Ahora bien, un dato que no puede pasar desapercibido es el hallazgo encontrado en el ítem denominado "Capacidad de cumplir con las fechas que los clientes requieren», resultó ser el de mayor impacto, obteniendo un resultado de 0.275, como puede observarse en la Tabla 2.

Con los resultados obtenidos a través de la regresión lineal, se obtiene la ecuación mostrada en la Figura 4.

Tabla 2. Variable de impacto en el modelo 1

Table 2. Variable Impact Model 1

\begin{tabular}{|c|c|c|c|}
\hline \multirow[t]{2}{*}{ Ítem } & \multicolumn{2}{|c|}{$\begin{array}{l}\text { Coeficientes no } \\
\text { estandarizados }\end{array}$} & \multirow{2}{*}{$\begin{array}{c}\text { Coeficientes } \\
\text { tipificados } \\
\text { Beta }\end{array}$} \\
\hline & B & Error típ. & \\
\hline Capacidad de cumplir con las fechas que los clientes requieren & 0.275 & 0.073 & 0.275 \\
\hline La flexibilidad en las necesidades cambiantes de los clientes & 0.218 & 0.068 & 0.218 \\
\hline $\begin{array}{l}\text { Interactuar con clientes para establecer estándares de fiabilidad y } \\
\text { capacidad de respuesta }\end{array}$ & 0.188 & 0.064 & 0.188 \\
\hline El tiempo de entrega de productos directamente a los clientes & 0.185 & 0.063 & 184 \\
\hline Adopción y prácticas de «Justo a Tiempo» & 0.132 & 0.062 & 0.133 \\
\hline
\end{tabular}

Fuente: elaboración propia. Datos obtenidos a partir del software SPSS versión 20. 
Rendimiento de la cadena de suministro $=0.268+0.275+$ Capacidad de cumplir con las fechas que los clientes requieren +.218 La flexibilidad en las necesidades cambiantes de los clientes + 0.188 Interactuar con clientes para establecer estándares de fiabilidad y capacidad de respuesta +0.185 El tiempo de entrega de productos directamente a los clientes +0.132 Adopción v prácticas de «Justo a Tiempo» + 0.073

Figura 4. Ecuación lineal del primer modelo

Figure 4. Linear equation of the first model Fuente: elaboración propia.

\section{Resultados del segundo modelo rendimiento y capacidad de agregar valor al servicio}

A continuación, en la Tabla 3 se muestran los resultados obtenidos en el segundo modelo, donde R obtuvo un valor de 0.164, una R2 de 0.027 y una R2 corregida de 0.022 , por lo que se establece que el clima organizacional se encuentra correlacionado con el desempeño en un $16.4 \%$, mientras que la influencia ejercida por parte del clima sobre el desempeño corresponde al $2.2 \%$, con lo cual a su vez se explica la estructura del modelo teórico, así mismo se puede concluir que la variable capacidad de generar valor al servicio influye en un porcentaje muy bajo.

Tabla 3. Resumen del modelo DesempeñoSatisfacción laboral

Table 3. Model Summary Job performanceSatisfaction

\begin{tabular}{lr}
\hline \multicolumn{1}{c}{ Modelo 1 } & \multicolumn{1}{c}{ Valores } \\
\hline R & 0.164 \\
R cuadrado & 0.027 \\
R cuadrado corregida & 0.022 \\
Error tip. de la estimación & 6.55578 \\
Durbin y Watson & 2.026 \\
Alfa de Cronbach & 0.887 \\
FIV & 1.000 \\
Significancia & 0.000 \\
\hline \multicolumn{2}{c}{ Fuente: elaboración propia. Datos obtenidos a } \\
\multicolumn{2}{r}{ partir del software SPSS versión 20. }
\end{tabular}

El análisis bivariado, efectuado mediante el cálculo de correlación de Pearson, arrojó en este segundo modelo asociaciones significativas en 4 de los 5 ítems considerados en la capacidad de generar valor sobre el rendimiento de la cadena de suministro. Las correlaciones que más se destacan en este apartado son: «Realizar servicios que agregan valor para el cliente durante el proceso de las ventas reales» ( $\beta=$ .149) $(P<.001 *)$, «Acomodo de pedidos especiales en el servicio al cliente». $(\beta=.146)(P$ $\left.<.001^{*}\right)$, «Adecuada introducción al mercado de los nuevos productos/servicios». $(\beta=.145)$ ( $P$ $\left.<.001^{*}\right)$, «Generar continuamente nuevos y variados productos» $(\beta=.156)(P<.001)$.

En el caso de este segundo modelo, el coeficiente alfa de Cronbach que se obtuvo como resultado fue de .887 , con lo cual se puede establecer que al igual que en el primer modelo, los niveles de fiabilidad del instrumento son altos y por ende están en un nivel óptimo para los propósitos de la investigación.

Ahora bien, en el caso de este segundo modelo también se logró un hallazgo en el ítem que cita textualmente "Realizar servicios que agregan valor para el cliente durante el proceso de las ventas reales», resultando ser el de mayor impacto, obteniendo un resultado de 0.032, como puede observarse en la Tabla 4. 
Tabla 4. Variable de impacto en el modelo 2

Table 4. Impact variable in model 2

\begin{tabular}{ccccc}
\hline \multirow{2}{*}{ Modelo } & & & Coeficientes no estandarizados & $\begin{array}{c}\text { Coeficientes } \\
\text { tipificados }\end{array}$ \\
\cline { 3 - 5 } & & B & Error típ. & Beta \\
\hline & $\begin{array}{l}\text { Realizar servicios agregan valor } \\
\text { cliente proceso de ventas reales }\end{array}$ & 0.032 & 0.518 & 0.164 \\
\hline
\end{tabular}

Fuente: elaboración propia. Datos obtenidos a partir del software SPSS versión 20.

Finalmente, en necesario hacer mención al resultado obtenido gracias a la técnica de la regresión lineal en este segundo modelo, la cual se muestra a continuación en la Figura 5.

\section{Desempeño $=3.934+0.032$ Realizar servicios que agregan valor para el cliente durante el} proceso de las ventas reales +0.518

Figura 5. Ecuación lineal del segundo modelo

Figure 5. Linear equation of the second model Fuente: elaboración propia.

Siguiendo con los resultados, y en lo que respecta a las hipótesis planteadas, se puede establecer que en relación con la $\mathrm{H} 1$ (la relación con los clientes es la variable que más influye al rendimiento de la cadena de suministro), se concluye que con base en los resultados previamente analizados la relación con los clientes, tiene una relación significativa y positiva sobre el rendimiento de la cadena de suministro, por lo que la $\mathrm{H} 1$ se acepta.

La H2 (a mejor relación con los clientes mayor rendimiento de la cadena de suministro) con base en los resultados y tomando en cuenta el resultado obtenido en la hipótesis 1 , se concluye que la $\mathrm{H} 2$ se acepta.

La H3 (las Pymes en Aguascalientes dan una gran importancia a la generación de valor en el servicio), con base en los resultados se puede establecer que las Pymes en Aguascalientes no toman como un factor importante la capacidad de generar valor al servicio por lo que la H3 se rechaza.
Finalmente, en lo que respecta a la H4 (Las Pymes en Aguascalientes dan una gran importancia a las relaciones con el cliente), se puede establecer que las Pymes en Aguascalientes dan una gran importancia a las relaciones con los clientes, por lo que la $\mathrm{H} 4$ se acepta.

\section{CONCLUSIONES}

Actualmente, y debido a las exigencias del mercado tan dinámico en el que subsisten las empresas, se vuelve un gran reto estudiar las variables que las organizaciones, y en especial las Pyme manufactureras de la ciudad de Aguascalientes, deben adoptar para mantenerse en un nivel competitivo en relación con las grandes compañías.

La ciudad de Aguascalientes, en los últimos años, ha sido una zona donde la Pymes manufactureras han podido proliferar, debido a la gran inversión que se ha hecho en el estado y a la llegada contante de empresas multinacionales, las cuales están ávidas de 
encontrar recursos para poder operar. Es ahí donde dichas Pymes deben aprovechar las oportunidades para establecer una cadena de suministro que las convierta en una opción factible y rentable para estas empresas, a través de la generación de valor y el establecimiento de relaciones.

Como conclusión de esta investigación, se puede decir que las relaciones con los clientes han demostrado ser una variable de alto impacto en el rendimiento de la cadena de suministro; por otro lado, aunque los resultados no muestren un gran impacto en la capacidad de crear valor sobre el rendimiento, hay que reconocer que en la literatura está probado que esta variable es tomada en cuenta como una de las más importantes para los clientes, es decir, las empresas deben de concentrar sus esfuerzos en conocer las necesidades de sus clientes con la intención de adoptar las medidas requeridas para cubrir los estándares de calidad. También es necesario crear algún tipo de recompensa para los clientes con lo cual se haga notar que todos los esfuerzos realizados añaden valor para ellos y sobre todo mantenerse en constante comunicación para evitar algún cabio o problema inesperado.

\section{REFERENCIAS}

Amid, A.; Ghodsypour, S and O'Brien, C. (2006). Fuzzy multiobjective linear model for supplier selection in a supply chain. International Journal of Production Economics, 104(2), 394-407.

Calderón H., G. (2006). La gestión humana y sus aportes a las organizaciones colombianas. Cuadernos de administración,19(31), 9-55.

Capó, J.; Exposito, M. y Masi, E. (2005). La gestión del conocimiento en las redes de Pymes. El caso del cluster textil valenciano. Revista de Economía industrial, 355, 305-315.
Capó-Vicedo, J.; Tomás-Miquel, J. V y ExpósitoLanga, M. (2007). La gestión del conocimiento en la cadena de suministro: análisis de la Influencia del contexto organizativo. Información Tecnológica, 18(1), 127-136.

Castro, B. y Hernández, S. C. (2008). La evaluación de las cadenas de valor como estrategia para la competitividad de las Pymes. Revista Tecsistecalt, (5).

Chopra, S. y Meindl, P. (2008). Administración de la cadena de suministro. Estrategia, planeación y operación, 3.

Correa, E. A y Gómez M., R. A. (2009). Tecnologías de la información en la cadena de suministro. Dyna, 76(157), 37-48.

Coyle, J. J.; Bardi, E. J. and Langley, Jr., C. J. (2003). The Management of Business Logistics. A Supply Chain Perspective. 7음 Edición. Mason, $\mathrm{OH}$ : Southwestern Publishing.

Chopra, Sunil y Meindl, P. (2008). Administración de la cadena de suministro. México: Pearson Prentice Hall.

García. S. F. (2006). La gestión de cadenas de suministros. Visión Gerencial, 5(1), 53-62.

Garrido M., A. (2008). La gestión de relaciones con clientes (CRM) como estrategia de negocio: desarrollo de un modelo de éxito y análisis empírico en el sector hotelero español. Servicio de Publicaciones de la Universidad de Málaga, España.

Giannakis, M.; Croom, S. R. (2004): Toward the Development of a supply chain management paradigm: A conceptual framework. Journal of Supply Chain Management, 40(2), 27-37.

Gómez, M. G. (2008). Cuantificación y generación de valor en la cadena de suministro extendida. Del Blanco Editores. 
El impacto de la relación con el cliente y de la capacidad de valor agregado en el servicio en el rendimiento de la PYME manufacturera

Gonzálvez G., N. (2016). Integración de los sistemas de información para la gestión de la cadena de suministro: un estudio empírico en grandes empresas (Doctoral dissertation), Universidad de Murcia). Recuperado de http://www.tdx.cat.dibpxy.uaa.mx/bitstrea m/handle/10803/370098/TNGG.pdf?seque nce $=1 \&$ iAllowed $=y$

Hair, J. F.; Black, W. C.; Babin, B. J.; Anderson, R. E. y Tatham, R. L. (2006). Multivariate data analysis (Vol. 6). Upper Saddle River, NJ: Pearson Prentice Hall.

Hashimura, H. (2011). Fundamentos para establecer una estrategia CRM.Redk. net REDK Software Engineering.

Jae-Eun, C. and Brenda, S. (2008). Japanese retail- buyer-supplier relationships: Does performance matter? Asia Pacific Journal of Marketing and Logistics, 20(1), 55-75.

Jiménez S., J. E. y Hernández G., S. (2002). Marco conceptual de la cadena de suministro: un nuevo enfoque logístico. Publicación Técnica, (215).

Luna, I. R. (2009). Pymes y cadenas de valor globales. Implicaciones para la política industrial en las economías en desarrollo. Análisis Económico, 24(57), 199.

Monge, E. C. (2010). Las estrategias competitivas y su importancia en la buena gestión de las empresas. Revista de Ciencias Económicas, 28(1).

Oly, N. (2005). Supplier selection and management strategies and manufacturing flexibility. Journal of Enterprise Information Management, 18(3), 330-349.

Sanchis, R.; Poler, R. y Ortiz, Á. (2009). Técnicas para el modelado de procesos de negocio en cadenas de suministro. Información tecnológica, 20(2), 29-40.

Saura, I. G.; Pérez, M. S.; Contrí, G. B. y GonzálezGallarda, M. (2006). Encuentro de servicio, valor percibido y satisfacción del cliente en la relación entre empresas. Cuadernos de Estudios Empresariales, (15), 47-72.

Sanchis, R.; Poler, R. y Ortiz, Á. (2009). Técnicas para el modelado de procesos de negocio en cadenas de suministro. Información tecnológica, 20(2), 29-40.

Torres S., M. C.; Escalante Ferrer, A. E.; Olivares Benítez, E.; Pérez García, J.C. (2016). Talento verde y cadenas de suministro verdes: ¿existe una relación significativa? Nova Scientia, 8(16), 421-454.

Young, R. y Esqueda, P. (2005). Vulnerabilidades de la cadena de suministros: consideraciones para el caso de América Latina. Academia. Revista Latinoamericana de Administración, 63-78. Recuperado de http://www.redalyc.org/articulo.oa?id=716 03405

Zutshi, A. and Creed, A. (2009). Child labour and supply chain: Profitability or (mis) management. European Business Review, 21(1), 42-63. 